\title{
Modeling exponential decay in maximum capacitance across specified flight patterns in small aircraft
}

\author{
Pete B. Rigas ${ }^{1}$, and Chetan S. Kulkarni ${ }^{2}$, \\ ${ }^{1}$ Cornell University, Ithaca, NY, 14850, USA \\ pbr43@cornell.edu \\ ${ }^{2}$ KBR, Inc., NASA Ames Research Center, Moffett Field, CA, 94035, USA \\ chetan.s.kulkarni@nasa.gov
}

\begin{abstract}
With increased autonomy being an integral part of unmanned aerial system (UAS), during flight a vehicle needs to have an accurate estimation of its state of health and capabilities to perform and achieve mission success with utmost safety. Batteries are of key importance in electric-propulsion aircraft and are its most pertinent resource. It is important to know the state of charge of the battery not only because the health state is directly related to the flight profiles flown by the vehicle, but also because the state of charge of the vehicle and its operational condition must be estimated after each flight.

In this work a methodology is presented to generate predictions for flight plans that experience anomalies, or unexpected system failure in due to a parasitic load in a specified stage of the flight that must return to its starting point of origin. We begin by describing the procedures by which a sequence of steps will be carried out to exponentially weigh the impact of different stages of a flight towards thermal strain on the capacitance $C_{\max }$ of the battery during each flight.
\end{abstract}

\section{INTRODUCTION}

Drawing from a previous 2014 work (Hogge \& et. al, 2014) in which the authors sought to improve methods of generating flight predictions for small aircraft, the methodology and approach that follows will generate flights predictions of a similar type, namely for flights with individual stages of specified duration, so as to improve the maintenance of batteries and other equipment that are involved in the aircraft. Moreover, to avoid having to perform laboratory tests on batteries after a fixed

Pete Rigas et al. This is an open-access article distributed under the terms of the Creative Commons Attribution 3.0 United States License, which permits unrestricted use, distribution, and reproduction in any medium, provided the original author and source are credited. number of flights within a cycle, the framework heavily relies on a free parameter choice to model the expected decay in battery capacity.

In contrast to the remaining flight time estimates that are put forth in (Hogge \& et. al, 2014), the approach is also aimed towards quantifying the exponential decay in the maximum capacitance due to a parasitic load that is inserted within a fixed stage of the flight, which can be imposed for the remaining duration of the flight. To categorically separate and study the rate of change of exponential decay of the maximum capacitance of different batteries, parasitic loads of exponential, or of polynomial, magnitude are computed for choices of $\beta$ parameters. From the percentage of the maximum capacitance that the battery is observed to hold after a given cycle, the exponential factor is temporally weighed in the duration of all stages of the flight. As a result, introducing this factor allows for a broader prognostics reflection not only pertaining to equipment maintenance but also to the total duration of a flight and the accompanying SOC before a failure occurs. Other works, including (Goebel \& Eklund, 2007),(Kulkarni et al., 2010),(Kulkarni et al., 2011),(Sankararaman \& Goebel, 2013a),(Sankararaman \& Goebel, 2013b),(Saha, Saha, \& Goebel, 2009) offer detailed discussions of either the experimental set up of tests which were used to test the accuracy of different numerical models, in addition to the architectural schemes of offline battery maintenance, insofar as to further study capacity degradation due to aging.

With example upper and lower bounds on the maximum capacity decay for a variety of flights from different batteries in the publicly available HIRF repository (Kulkarni et al., 2015), we are interested in building upon previous studies to further study the capacitance degradation. The publicly available data set contains HIRF tests and experimental data conducted on an electric fixed wind UAV Edge 540. The e-UAV is a $33 \%$ sub- 
scale version of the Zivko Aeronautics Inc. Edge $540 \mathrm{~T}$ tandem seat aerobatic aircraft. This vehicle has been actively used to facilitate the rapid deployment and evaluation of remaining flying time prediction algorithms for electric aircraft(Hogge \& et. al, 2014).

The parameter choice and resulting numerical behavior provides a formalism through which experiments consisting of flights with either longer duration, more complicated pattern with more stages in the flight, or varying magnitude of electromagnetic radiation in the ambient environment, can be accounted for through adjustment of the free parameters.

To characterize different flight plans from which sets of predictions will be generated, the approach detailed below provides approximations of the time duration of each segment of a flight, depending on the magnitude of the current exerted by the engine over each time interval. To readily generate valuable prognostics predictions, we introduce an exponentially decaying factor whose magnitude is numerically determined by enforcing choices of parameters $\left\{\beta_{i}\right\}$ which multiply the approximate duration of each time interval of the flight. Finally, with a smooth polynomial approximation to the flight data, we compute the Fourier coefficients and series of current versus time plots of a given flight, to generate predictions for upcoming flight plans. From batteries of a given type, the magnitude of the exponential factor will be leveraged to model the decay in the maximum capacitance of the battery across multiple flights.

\section{General Approach}

In this section an overview of the developed approach is discussed. The Fourier series representation of each flight plan is obtained by approximating the temporal duration of each segment of an arbitrary flight plan through each stage of the flight, which includes imposing a coordinate axis to measure applicable sine or cosine modes of the Fourier series representation which will be enforced after coefficients corresponding to each stage of the flight have been determined.

Next, we compute a sufficient number of Fourier coefficients so that the corresponding series representation of the flight plan sufficiently represents current measurements for each point in time of the flight plane, which is obtained through a trigonometric basis $\left\{\sin \left(\frac{n \omega_{0} t}{T}\right), \cos \left(\frac{n \omega_{0} t}{T}\right)\right\}_{n \in \mathbf{N}}$, where a normalizing factor $T$ is introduced.

For one example flight, we determine a threshold of the Fourier modes $\mathcal{N}$ for which the mean squared error, of the form $\sum_{n} a_{n}^{2}+b_{n}^{2}$, or equivalently $\sum_{n} a_{n}^{2}$ or $\sum_{n} b_{n}^{2}$, is determined. Across numerical experiments in which up to 20,000 Fourier modes are computed, we conclude that the approach is computationally accessible because a MSE of magnitude $\approx 0.192$ demonstrates that the methodology is capable of generating reliable predictions for varying flight profiles without having to include hundreds of Fourier modes (in particular, no more than 20 from the worked example in Section 4.2) in the series expansion. From either a Fourier cosine or sine series, informative and reliable predictions can be generated by determining the final time stamp up to which the polynomial approximation of flight data will be enforced.

Next, given such a Fourier series representation of the flight, the exponentially decaying factor for computing the change in the maximum and minimum capacitance will be defined. In turn, the approach will generate predictions for flights plans of the following types, all of which are dependent on the duration of the specific stage of the flight as well as the time stamp throughout the stage of the flight at which the plan is terminated. It is important to emphasize that the time stamp at which the flight will be terminated is arbitrary and will therefore impact the corresponding number of Fourier modes and MSE that can be computed. In what follows, we will distinguish between different flight plans, depending on the number of stages in a flight, at which the flight can be arbitrarily terminated.

Flight Plan Type A: Given a set of experimental trials, we will demonstrate how to compute an accompanying rate of exponential decay in the maximum capacitance over successive flights that is proportional to the magnitude, and duration, of the thermal stress in addition to the mechanical work that is applied to the engine for each stage of the flight. From this factor, predictions will be readily generated for flights that are terminated in the first stage of the flight by computing the exponential decay of the maximum capacitance $C_{\max }$. In addition to examining the sharpness of decay in $C_{\max }$, our methodology is also capable of accommodating different series expansions, namely expressions with a different number of Fourier modes $\mathcal{N}$ that can be used to accommodate predictions for different time stamps throughout some fixed stage of the flight. Within a given stage of the flight, the smooth polynomial approximation of flight data is capable of generating distinct Fourier series expansions, each of which correspond to flight plans in which the rightmost endpoint in time is continuously varied. With variable time stamps at which the flight is terminated, the polynomial root finding method generates smooth approximations from experimental current versus time plots.

Flight Plan Type B: Given a set of experimental trials, predictions for more complicated flight patterns can be generated by making use of the predictions generated for flights of the previously defined type, in the sense that 
a flight pattern of Type B can be analyzed, from predictions of fights of Type A by implementing the following. First, it is imperative that we include a prescribed number Fourier modes $\mathcal{N}$ in the series expansion for the beginning phase of the flight during which current flows through the circuit. In this initial stage, predictions of the termination of the flight plan in the next stage of the flight, hence the name Flight Plans of Type B, can be generated by implementing the change in maximum capacitance from the previous flight plan. From the free parameters $\beta_{i}$ that we have injected into the power of the exponent to measure the decay in $C_{\max }$, we can also generate sets of inequalities to capture the minimum and maximum rates of exponential decay in $C_{\max }$, granted that the smooth polynomial approximation not violate the condition that $\left|R\left(t_{i}\right)-f\left(t_{i}\right)\right|<\epsilon$ for all times $t_{i}$ preceding the unforseen abortion of the flight in its earlier stage. In this inequality, the precision that we demand between the smooth polynomial approximation of the flight plan data and the current measurements holds for arbitrary $\epsilon$, where the quantity $R$ denotes the experimentally gathered flight plane for all time stamps $t_{i}$ in the flight, and the quantity $f$ denotes the smooth polynomial approximation of the current measurements. When running the polynomial root finding algorithm, we enforce that a maximum discrepancy $\epsilon$ between the experimental data, and approximations of the data, is satisfied across all $t_{i}$.

Flight Plans Types C,D,E: Repeating the same observations and formulations for flight plants of Type A and Type B mutatis mutandis gives immediate generalizations of other flight plans for which the unforseen abortion of the flight can occur. In particular, flight plans of Types C, D and E, respectively, describe excursions over which the same maximum capacitance value (which is the $C_{\max }$ value computed from previous flights in a given sequence, determined from an initial capacitance value in the first flight of the sequence which we set to be at a $100 \%$ threshold), the final time stamp $t_{f}$ are which the flight is terminated.

Flight Plan Type X: Pursuant of further generalizations, the preceding observations and formalism can be applied mutatis mutandis to new flights patterns, of emerging interest, for an arbitrary but countable number of intervals over which a flight can be terminated.

The authors suggest that generalizing the approach to new experiments with small aircraft of varying construction and battery types is worthwhile. The publicaly available dataset from NASA includes collections of current time measurements, which can be modeled with the following approach by roughly determining the duration of each period of the flight, from which additional flight tra-

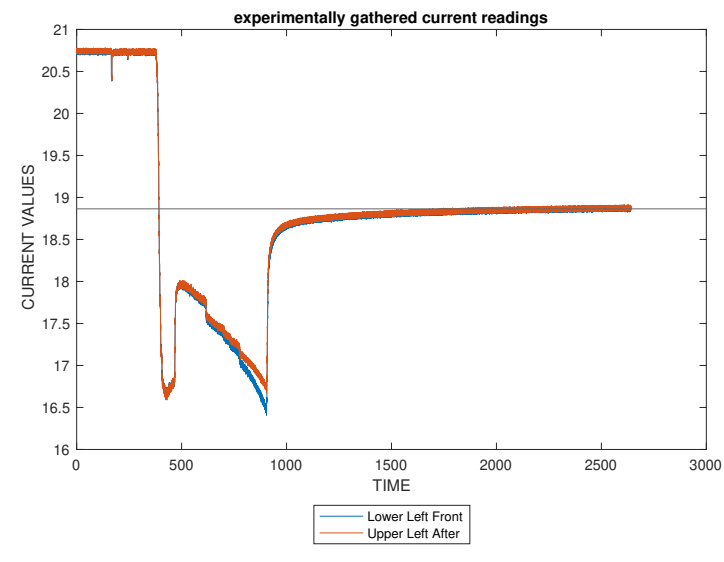

Figure 1. Example of Set 6, HIRF 77, plot of horizontal line for flight plan of Case 1

jectories rather than those carried out by experiment can be simulated with varying free parameter choices.

\section{IMPLEMENTATION}

To test the Fourier series implementation and exponentially decaying capacitance factor in flight plans, we implement the approach in a set of training data through a given sequence of flights by executing the following.

We run an auxiliary algorithm in Matlab which returns the approximate time intervals, and respective duration, of each stage of a given flight. Systematically, we deal with the following cases.

Case 1: (Flight plans in which the current reading at the final stage of the flight vertically asymptotes to a current measurement higher than that of preceding stages of the flight) For flight plans of Case 1, one can easily generate time intervals for intermediate stages of the flight plan by extending a horizontal line past the stage of the flight, and then linearly searching below a determined threshold to obtain isolated segments of the flight during which polynomial approximations from the root finding method can be obtained. From the current value that is exerted near the end of the flight from which the horizontal line is extended, a threshold $\delta$ will be chosen so that all current values below those of $\mathcal{H}-\delta$ are returned.

Case 2: (Flight plans in which the final current reading for the last stage of the flight vertically asymptotes to a value that is lower than that of the preceding stage of the flight) For flight plans of Case 2, one can generate analogous results corresponding to the time intervals for disjoint segments of a given flight plan. For Case 2, we instead linearly search above the current value that the data asymptotically approaches at the final time 


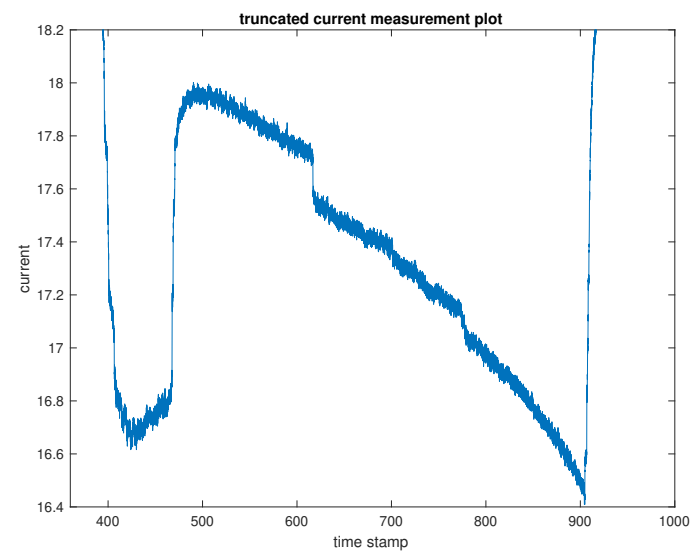

Figure 2. Plot of individual stages of the flight which are determined by linear inspection of the data through a specified threshold $\delta$ of the current asymptotic value given by the horizontal line at the end of the flight, with $\delta=-0.5$.

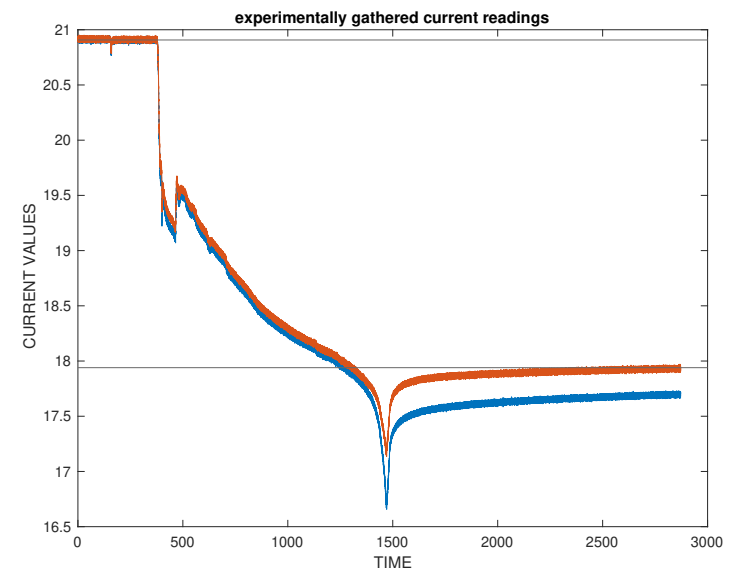

Figure 3. Example of Set 6, HIRF 80, plot of two horizontal lines for flight plans falling under Case 2

stamp of the given flight, while simultaneously conditioning that the search enforce that the isolated segments of the flight not exceed the initial current starting value in the initial stages of the flight. After executing such a search, the auxiliary algorithm similarly returns a set of times for each stage of the flight.

With the time duration of each stage, the resulting values inform the magnitude of an exponentially decaying factor which measures the change in $C_{\max }$ value across subsequent flights. This temporally weighed factor is of central importance for the methodology because the exponential factor informs the sharpness of capacitance decay which is inversely proportional to the total flight time. From the flight examples above, we observe the following.

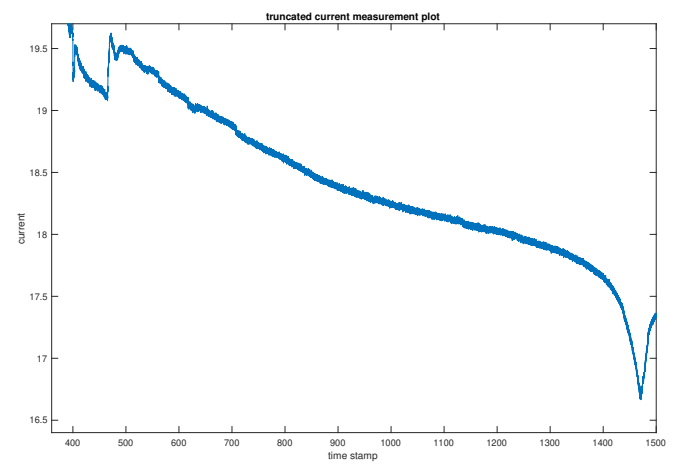

Figure 4. Plot of individual stages of the flight which are determined by linear inspection of the data through a specified threshold $\delta^{\prime}$ of the current asymptotic value given by the horizontal line at the end of the flight, with $\delta=1.5$.

\begin{tabular}{|c|c|c|}
\hline Case & Parameters & $C_{\max }$ on flight 2 \\
\hline \hline 1 & $\beta_{1}=\frac{1}{500}, \beta_{2}=\frac{1}{5000}$ & $\approx 0.73184 C_{1}$ \\
\hline 2 & $\beta_{1}=\frac{1}{100}, \beta_{2}=\frac{1}{5000}$ & $\approx 0.43509 C_{1}$ \\
\hline 3 & $\beta_{1}=\frac{1}{2500}=\beta_{2}$ & $\approx 0.67686 C_{1}$ \\
\hline 4 & $\beta_{1}=\frac{1}{25000}=\beta_{2}$ & $\approx 0.99610 C_{1}$ \\
\hline
\end{tabular}

Table 1. Table providing choice of parameters for each of the 4 cases in Figure 5

From Set 6, HIRF 77, disregarding the middle portion of the flight during which the magnitude of the loading current is approximately constant, one may compute the exponential decay in $C_{\max }$ by enforcing choice of free $\beta_{i}$. In this specific instance, the choice of free parameters correspond to the real numbers that are linearly weighed in the temporal duration of all segments of the flight. Upon drawing inspiration from exponential solutions to the one dimensional separable differential equation, we expect that comparing the temporal duration of different stages of a flight, from the example in Set 6, HIRF 77 above, yields an exponential decay $C_{\max }$ that would further reduce the change in capacitance in the second flight. It is natural to expect that the rate of capacitance decay will decay more sharply if there are more time stamps in a given stage of the flight, in which $C_{\max _{1}}^{1 \rightarrow 2} \geq C_{\max _{2}}^{1 \rightarrow 2} \Leftrightarrow$ $\left|T_{6,77}^{1}\right|=\left|\left\{t_{i} \in T_{6,77}^{1}\right\}\right| \leq\left|T_{6,77}^{2}\right|=\left|\left\{t_{j} \in T_{6,77}^{2}\right\}\right|$. The ordered pair $\left(C_{\max _{2}}, T^{2}\right)$ denotes the capacitance and stage duration, respectively, of the stage $T_{6,77}^{1}$ of an arbitrary flight plan.

On the other hand, from Set 6, HIRF 80, the return values of the auxiliary algorithm permit calculations of the exponential decay in $C_{\max }$ for flights of Case 2. From noticeable differences in the trajectory of the flight from 


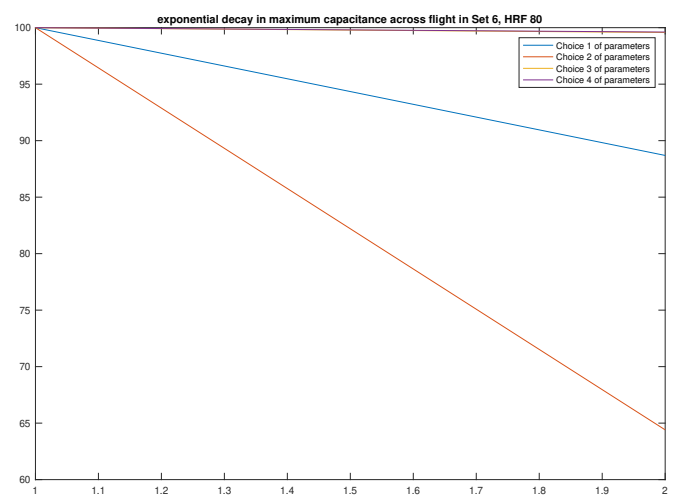

Figure 5. From the two demarcated stages of the flight given the return values of the auxiliary algorithm, the exponential decay in $C_{\max }$ is exhibited across each stage of the flight. A clear dependence between the reported $C_{\max }$ and $\beta_{i}$ is exhibited.

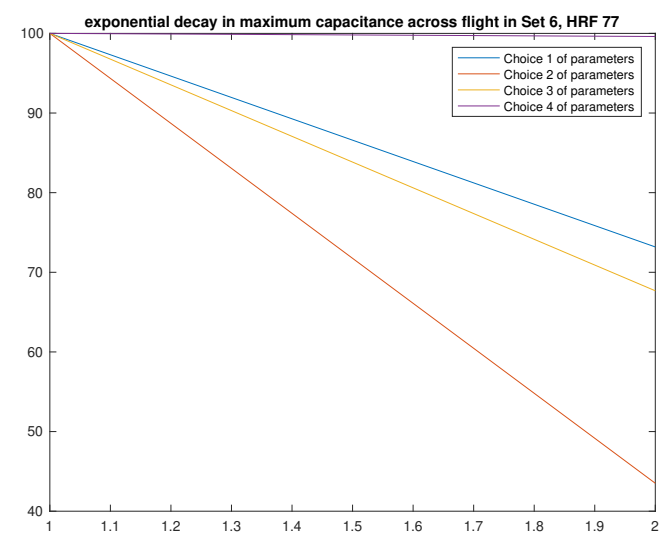

Figure 6. From the two demarcated stages of the flight given the return values of the auxiliary algorithm, the exponential decay in $C_{\max }$ is shown across the first stage of the flight.

Set 6 , the exponential decay in $C_{\max }$ is more sharp across the sequence of flights in comparison to that of HIRF 80.

Finally, to establish sets of predictions for flight of varying types, namely flights of the previously defined types, a Fourier series expansion will be introduced by computing the Fourier coefficients from the polynomial approximation.

\subsection{Exponentially decaying capacitance}

With the different choices of $\beta$ parameters that will be further expounded upon in more sensitive calculations of the exponential decay in $C_{\max }$ that are inclusive of the beginning and ending periods of the flight, we will make the choice of free $\beta$ parameters so that the decay of the maximum capacitance between neighboring flights

\begin{tabular}{|c|c|c|}
\hline Case & Parameters & $C_{\max }$ after flight 2 \\
\hline \hline 1 & $\beta_{1}=\frac{1}{1000}, \beta_{2}=\frac{1}{5000}$ & $\approx 0.88692 C_{1}$ \\
\hline 2 & $\beta_{1}=\frac{1}{200}, \beta_{2}=\frac{1}{5000}$ & $\approx 0.644036 C_{1}$ \\
\hline 3 & $\beta_{1}=\frac{1}{300000}, \beta_{2}=\frac{1}{50000}$ & $\approx 0.995742 C_{1}$ \\
\hline 4 & $\beta_{1}=\frac{1}{300}=\beta_{2}$ & $\approx 0.99610 C_{1}$ \\
\hline
\end{tabular}

Table 2. Table providing choice of parameters for each of the 4 cases in Figure 7.

reflects a rate of decay in the capacitance that is appropriate, in the sense that the rate of exponential decay does not fall into any one of the cases above in which the maximum capacitance in one flight from a previous one is almost left unchanged, at or around $99 \%$ of the previous $C_{\max }$, or at around $50 \%$ of the $C_{\max }$ for a typical flight.

\section{WORKFLOW}

Before proceeding with our formalism to define quantities that are of computational value to help with generating flight predictions, the items below provides a high level summary of each step in the approach.

1. The auxiliary algorithm uses flight plan data as input,which returns the approximate time intervals of each stage of the flight, depending on the magnitude of the current at the beginning and ending stages of the flight.

2. We enforce choices of free $\left\{\beta_{i}\right\}_{i \in \mathbf{N}}$ for calculating the exponential decay of $C_{\max }$.

3. With lower and upper bounds on such a decay rate, polynomials are introduced to smoothly approximate chamber data for current measurements in the aircraft engine, from which estimates of each stage of the flight are generated.

4. To approximate flight plan data with a Fourier series, smooth polynomial approximation is used on the flight data obtained in the previous step, as we compute the sine and cosine coefficients of the Fourier series expansion.

5. As a result, sets of predictions for future flights are generated, within the combinatorial space of distinct outcomes, by computing the range of Fourier modes that can be used in the series to approximate the flight plan data. With the Fourier coefficients of our expansion, we can also determined corresponding MSE values. From numerical experiments on data from the NASA prognostics repository, we are interested in determining whether there is a critical value of the Fourier modes, $\mathcal{F}_{\text {crit }}$, beyond which increasing the number of Fourier modes in the series expan- 
sion does not yield a lower MSE. Qualitatively, we attribute a lower MSE to a higher confidence in the set of predictions for a flight on which the algorithm is ran, while we attribute a higher MSE to a lower confidence in the set of predictions.

6. Within the space of outcomes, we will introduce numerical bounds, based on the $30 \%$ SOC threshold cutoff, at which a flight can safely operate for a specified duration of time.

\subsection{Fourier series approach}

With a polynomial approximation obtained via the root finding method, given an arbitrary number of time stamps before the flight plan is to be terminated, a modification to the usual Fourier sine and cosine coefficients will be introduced with each of the coefficients taking the for

$$
a_{n}=\frac{1}{\sum_{i} t_{i}} \int_{\mathcal{T}_{i}} f(t) \cos \left(\frac{n \omega_{0} t}{\mathcal{T}_{i}}\right) \mathrm{d} t,
$$

and

$$
b_{n}=\frac{1}{\sum_{i} t_{i}} \int_{\mathcal{T}_{i}} f(t) \sin \left(\frac{n \omega_{0} t}{\mathcal{T}_{i}}\right) \mathrm{d} t,
$$

where $\mathcal{T}$ denotes the stage of the flight over which the polynomial approximation is obtained, and the normalizing constant for the period in the Fourier coefficients is the summation over all time stamps of the particular stage of the flight. As expected, $f$ is smooth and from these expressions, one can obtain a series expansion of the form

$$
F(x)=\frac{a_{0}}{2}+\sum_{n, t \in \mathcal{T}_{i}} a_{n} \cos \left(\frac{n \omega_{0} t}{\mathcal{T}_{i}}\right)+b_{n} \sin \left(\frac{n \omega_{0} t}{\mathcal{T}_{i}}\right),
$$

where each period $\mathcal{T}_{i}$ is not only dependent on the duration of the time interval of the stage of the flight, but also on the current of the magnitude $\mathcal{I}$ exerted on the engine given a trajectory. With such a Fourier series that one can obtained from the aforementioned trigonometric basis, introduced over each respective $\mathcal{T}_{i}$, flight predictions for plans of ype A can be generated for arbitrary time stamps $t_{j}$ before the initial stage of the flight by first introducing a polynomial approximation of the flight data up to $t_{j}$, from which the MSE of all modes included in the expansion, $\sum_{n} a_{n}^{2}+b_{n}^{2}$, is computed. Up to the stage of interest, we make use of the truncated Fourier series,

$$
F_{\mathcal{T}_{i}, t_{i}}=\frac{a_{0}}{2}+\sum_{n=1}^{\mathcal{M}_{t_{i}}} a_{n} \cos \left(\frac{n \omega_{0} t}{\mathcal{T}_{i}}\right)+b_{n} \sin \left(\frac{n \omega_{0} t}{\mathcal{T}_{i}}\right),
$$

for a sufficient number of modes $\mathcal{M}_{t_{j}}<\mathcal{N}$. With a series approximation $F_{\mathcal{T}_{i}, t_{i}}$ above denoting the series truncation corresponding to the maximum time stamp $t_{i}<t_{j}$ for which the $30 \%$ SOC threshold can be maintained when the flight is terminated at the later time $t_{j}$, for all $t \in \mathcal{T}_{i}$.

To ensure that such a truncation adequately captures the degradation in $C_{\max }$ across successive trials, or potentially across trials with a battery of any type, an exponentially decaying, temporally dependent factor, factor is defined across all time stamps $t_{i}, t_{j} \in \mathcal{T}_{i}$, and $t_{i}^{\prime}, t_{j}^{\prime} \in \mathcal{T}_{j}$. Free parameters $\beta_{1}, \beta_{2}$, as demonstrated in plots for different decay rates of $C_{\max }$, which multiplicatively accompany the time intervals $\left[t_{i}, t_{f}\right]$ and $\left[t_{i}^{\prime}, t_{f}^{\prime}\right]$, over $\mathcal{T}_{i}$ and $\mathcal{T}_{j}$, respectively, during which a current of varying magnitude $\mathcal{I}$ is exerted. More generally, the power of the exponent is of the form $\sum_{i} \beta_{i}\left|\mathcal{T}_{i}\right|$, with the summation taken over all stages of the flight.

For distinct time periods of each stage, the rates of exponential decay in $C_{\max }$, in addition to the series representation, provide flight predictions by computing the rate of exponential decay in the $C_{\max }$ across multiple flights with specified duration from the periods $\mathcal{T}_{1}^{1}, \cdots, \mathcal{T}_{n}^{1}, \cdots, \mathcal{T}_{N}^{1}, \cdots, \mathcal{T}_{N}^{n}$. Incorporating the exponential decay mentioned in the previous step to determine the maximum duration's of time for which the flight can maintain the $30 \%$ threshold, can be obtained by making use of the formula

$$
\mathrm{SOC}=1-\frac{q_{\max }-q_{b}}{C_{\max }},
$$

provided for computing the threshold in [2]. With the previous exponential factor, it is also possible to determine the range of admissible Fourier modes in the series representation and to compute the MSE associated with the series approximation.

\subsection{Computation Case Studies}

With a substantial array of Fourier coefficients, ranging from 200, 2, 000 to 10,000 modes, all of which together constitute a run time of approximately 3 days, numerical simulations were executed to determine properties relat- 


\begin{tabular}{|c|c|c|}
\hline Fourier modes & Max & Min \\
\hline \hline 200 & $\approx 0.40793$ & $\approx 2.7658 \times 10^{-6}$ \\
\hline 2,000 & $\approx 0.40793$ & $\approx 2.7049 \times 10^{-8}$ \\
\hline 10,000 & $\approx 0.40793$ & $\approx 1.0955 \times 10^{-3}$ \\
\hline
\end{tabular}

Table 3. Fourier cosine coefficients

\begin{tabular}{|c|c|c|}
\hline Fourier modes & Max & Min \\
\hline \hline 200 & $\approx-8.6023 \times 10^{-4}$ & $\approx-21.1558$ \\
\hline 2,000 & $\approx-8.6023 \times 10^{-5}$ & $\approx-21.1558$ \\
\hline 10,000 & $\approx-1.7205 \times 10^{-5}$ & $\approx-21.1558$ \\
\hline
\end{tabular}

Table 4. Fourier sine coefficients

ing to the convergence of Fourier series, which is valuable for determining the critical number $\mathcal{F}_{\text {crit }}$. By introducing a series approximation to the end of the first stage of this flight up to $t_{f}=450$, the numerical experiments demonstrate that we do not have to resort to a high number of Fourier modes in order to reach a sufficiently low MSE. This demonstrates that utility of the approach in being able to readily generate predictions that are computationally accessible.

Beyond observed numerical results, the plots below exhibit the distribution of higher frequency terms that could be used in the Fourier series expansion, where the computations of the Fourier coefficients are carried out for 10, 000 Fourier sine and cosine coefficients. Due to the significantly higher MSE associated with the Fourier sine coefficients for this example flight, we have only made use of the cosine coefficients in the series expansion to approximate the flight data, from which the series expansion is plotted for all 4938 time stamps of the flight.

\subsection{Adjusting the free parameters in the exponen- tially decaying factor}

From the description and implementation of the auxiliary algorithm as a proxy for the change in $C_{\max }$ across a flight, from a fixed ordering of the time stamps and current measurements, the image of time intervals under the

\begin{tabular}{|c|c|}
\hline Fourier modes & MSE \\
\hline \hline 200 & $\approx 0.192276077317043$ \\
\hline 10,000 & $\approx 0.192276132208228$ \\
\hline
\end{tabular}

Table 5. MSE values

\begin{tabular}{|c|c|c|}
\hline Fourier modes & Max. & Min. \\
\hline \hline 200 & $\approx 0.3139$ & $\approx 7.928 \times 10^{-6}$ \\
\hline 2,000 & $\approx 0.3139$ & $\approx 7.857 \times 10^{-8}$ \\
\hline
\end{tabular}

Table 6. Fourier cosine coefficients

\begin{tabular}{|c|c|c|}
\hline Fourier modes & Max. & Max. \\
\hline \hline 200 & $\approx 21.124$ & $\approx 2.466 \times 10^{-3}$ \\
\hline 2,000 & $\approx 21.236$ & $\approx 2.466 \times 10^{-4}$ \\
\hline
\end{tabular}

Table 7. Fourier sine coefficients

assignment $\Phi: \mathbf{R}^{+} \longrightarrow \mathbf{R}^{+}:\left|t_{j}-t_{k}\right| \mapsto \beta_{i}$, for $j \neq k$, and $\beta_{i}>0$, produces a monotonic pairing of the time intervals of each stage of the flight with the freely chosen $\beta_{i}$ so that, throughout the variable duration of a given flight pattern, periods of shorter duration over which the magnitude of the current $\mathcal{I}$ abruptly changes are multiplicatively weighed unfavorably in their negative impact on the ability of the circuit to transport charge in the engine, while periods of longer duration over which the current of magnitude $\mathcal{I}$ changes more gradually over the course of a stage in the flight, are multiplicatively weighed favorably in terms of continuing to preserve the ability of the circuit to maintain the maximum capacitance which in turn less adversely impacts the circuit.

With such a convention of the free parameter assignment, a range of exponentially decaying factors, allows for predictions to be realized. Through the experiment with the arrays of Fourier coefficients that have been generated, the same approach can be applied to flight patterns for which the time intervals of each stage are collected, from which the temporally adjusted weighted exponential decrease of the circuit can be computed which is dependent on the magnitude of the current exerted during stages of the flight. Such data sets are publicly available from the NASA prognostics repository 15.

\begin{tabular}{|c|c|}
\hline Fourier modes & MSE \\
\hline \hline 200 & $\approx 560.79416$ \\
\hline 2,000 & $\approx 561.902567337$ \\
\hline 10,000 & $\approx 5.62001093$ \\
\hline
\end{tabular}

Table 8. MSE values 

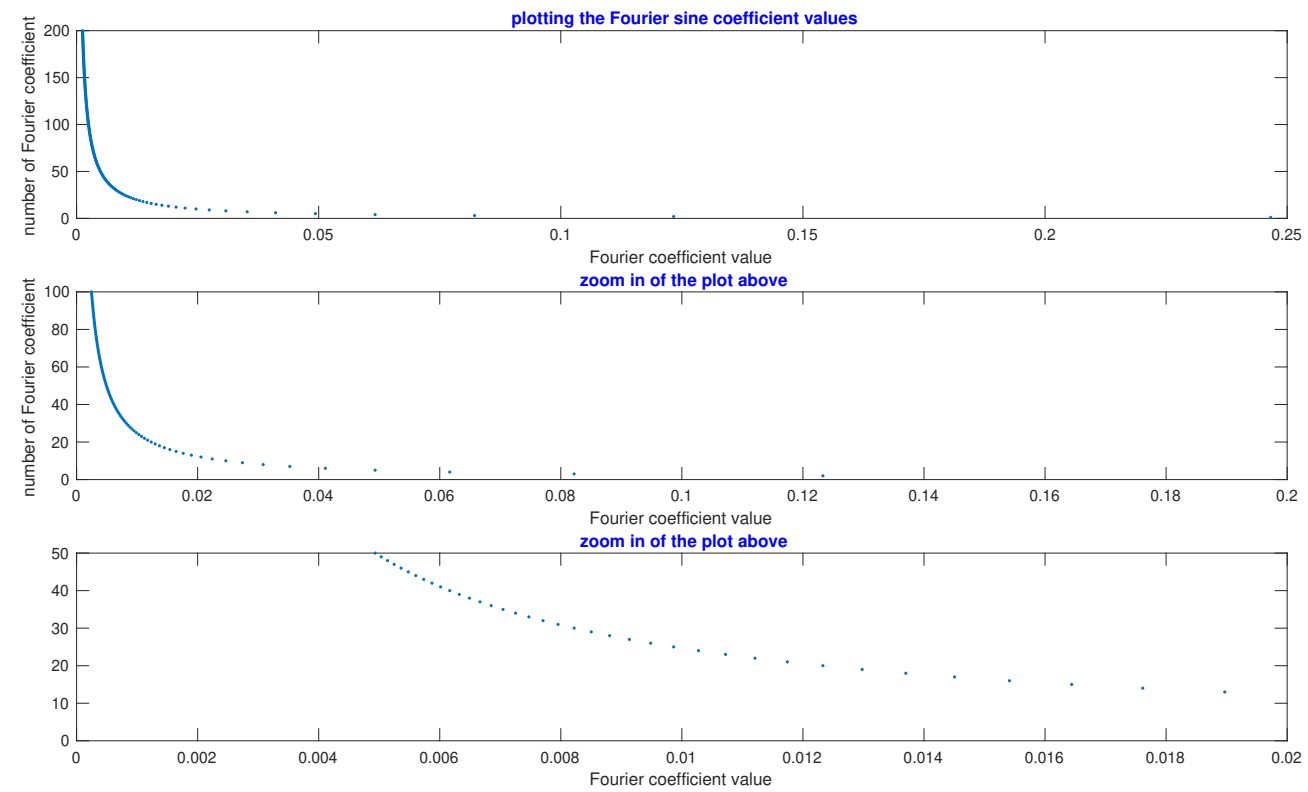

Figure 7. In contrast to similar plots of the Fourier cosine coefficients, the sine coefficients, for the truncated polynomial approximation up to the time stamp 450 before the current change occurs results in coefficients that are of opposite sine than those previously presented in the previous diagram.

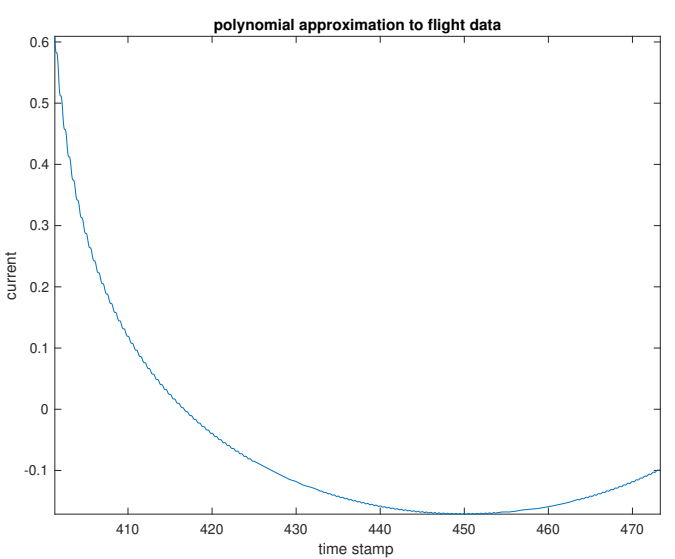

Figure 8 . To more closely analyze the behavior of the series approximation from which predictions will be generated with a corresponding MSE, the behavior of the series approximation, as $t$ varies, is depicted above.

\section{Procedure for generating the combina- TORIAL SPACE OF FLIGHT PREDICTIONS}

To produce effective and informative flight predictions given a numerically specified exponential rate of decay in $C_{\max }$, we proceed with the following.

Step 1 (identifying the stage of the flight during which the prediction is to be established): To systematically address all stages and times during which an anomaly could occur, we introduce arbitrary time stamps $t_{\text {abort }}^{(i)}$ corresponding to each stamp $T^{(i)}$ of a given flight. From such time stamps in future flight plans that we attribute to anomalies, predictions for the flight in question will be generated by linearly varying the stamp $t_{\text {abort }}^{(i)}$ with respect to time for all admissible stamps in the stage $\mathcal{T}^{(i)}$ of the flight. From such a collection of times, we proceed to incorporate the minimum and maximum rates of exponential decay in $C_{\max }$ of the circuit, by means of introducing a suitable free parameters $\beta_{i}$ in the exponentially decaying factor, insofar as to not only simulate distinct rates of exponential decrease in $C_{\max }$, but also to account for flight plan anomalies in any possible stage. To determine whether a flight should be terminated, at arbitrary $t_{\mathrm{SOC}}^{(i)}<t_{\text {abort }}^{(i)}$, we make use of the expression for the state of charge threshold given in [2].

Step 2 (computing informative ranges in the exponential decay of $C_{\max }$ ): In order to generate realistic predictions, we must also introduce upper and lower bounds for the decay of $C_{\max }$. Pursuant of this goal, we wish to measure the change in exponential decay that one can attribute to different choices of parameters $\beta_{j}$, denoted $\beta_{j}^{\prime}$, satisfying $\left|\beta_{j}-\beta_{j}^{\prime}\right|<\epsilon$ for arbitrarily small $\epsilon$. Under these assumptions, the exponential factor for measuring the change in capacitance takes the form, 


$$
\overbrace{\overbrace{\left.e^{\sum_{i \cap j} \beta_{i}\left(t_{\text {final }}^{(i)}-t_{\text {initial }}^{(i)}\right)+\beta_{j}^{\prime}\left(t_{\text {final }}^{(i)}-t_{\text {finitial }}^{(i)}-t_{\text {initial }}^{(j)}\right)+\beta_{j}\left(t_{\text {final }}^{(j)}-t_{\text {initial }}^{(j)}\right)}\right)}^{\text {for first choice of } \beta_{j}},}^{\text {for second choice of } \beta_{j}},
$$

where the arbitrary reals $\beta_{j}, \beta_{j}^{\prime}$ are chosen during the stage $T^{(j)}$ of the flight, which has time duration $t_{\text {final }}^{(j)}-$ $t_{\text {initial }}^{(j)}$. Because all of the remaining $\beta_{i}$, for $i \neq j$ for all other stages of the flight are fixed, enforcing a different choice of the $\beta_{j}$ parameter amounts to a multiplicative, exponentially decaying factor, of the form

$$
\left.e^{\sum_{i \cap j} \beta_{i}\left(t_{\text {final }}^{(i)}-t_{\text {initial }}^{(i)}\right.}\right)\left(e^{\left(\beta_{j}-\beta_{j}^{\prime}\right)\left(t_{\text {final }}^{(i)}-t_{\text {initial }}^{(i)}\right)}\right) .
$$

Additionally, to make even more explicit the dependence of the $C_{\max }$ decay with respect to time, we designate the arbitrary time stamps $t_{\text {abort }}^{(i)}$ as the times during each stage $\mathcal{T}^{(i)}$ during which the flight could be terminated. At each such time stamp in this stage, the choice of parameters will satisfy the SOC threshold that is provided in future arguments.

Step 3 (choosing flight data from the prognostics repository to compute exponential rates of decay in $C_{\max }$, from which bounds on the $C_{\max }$ will be generated: From previous remarks concerning how the $C_{\max }$ of the circuit would change across successive flights with choices of $\beta_{i}$ for each flight stage, as a consequence it is important to designate the sets of experimental data from which exponential rates of decay in $C_{\max }$ will be determined. In addition to enforcing a certain SOC threshold that the aircraft must satisfy while it is operational, predictions for future flights can be generated by determining appropriate choices of the $\beta_{i}$ so that the model reliably captures the rate of $C_{\max }$ as observed from experiments.

Step 4 (generalizing sets of predictions for flight plan

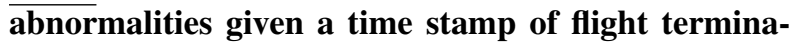
tion): With upper and lower bounds for $C_{\max }$ in hand from Step 3, generating flight predictions now amounts to implementing the variable time, to be specified between stages of a flight, during which an arbitrary flight plan could be aborted due to either flight plan abnormalities or mechanical failures. In particular, by choosing each possible stage of the future flight plans at which the anomaly could occur, predictions are readily generated by searching for parameters that capture the rate of decay in the maximum capacitance.

In specific instances of different flight planes, the approach is capable of determining whether a small aircraft can land given unforseen changes in the $C_{\max }$ value of the circuit. With an additional exponentially decaying factor appended to the quantity has has already been given and discussed, which takes the form

$$
\begin{gathered}
\mathcal{C}_{\max _{i+1}}^{\mathrm{PL}, T^{(i)}}=\left(e^{\sum_{i}-\beta_{i}\left|t_{\text {initial }}^{(i)}-t_{\text {final }}^{(i)}\right|} \times\right. \\
\left.e^{\beta^{\mathrm{PL}, \mathcal{T}^{(i)}}\left|t_{\mathrm{final}}^{\text {PL }}-t_{\text {initial }}^{P L}\right|}\right) C_{\max _{i}},
\end{gathered}
$$

which denotes the local exponential decay of $C_{\max }$ given a temporally dependent, exponentially increasing, parasitic load PL. Numerically, it is important to remark upon the nature of this exponential term, particularly in that the power of the exponential can be equal to, less than, or greater than in magnitude of the summation in the power of the first exponential which is dependent on both the $\beta_{i}$ and duration of each stage $\mathcal{T}^{(i)}$ of the flight. One may also define the decay of the local capacitance due to a polynomial factor, instead of an exponential one, taking the form

$$
\begin{gathered}
\mathcal{C}_{\max _{i+1}^{\mathrm{PL}, T^{(i)}}}=\left(e^{\sum_{i}-\beta_{i}\left|t_{\text {initial }}^{(i)}-t_{\text {final }}^{(i)}\right|} \times(1\right. \\
+\left(\beta_{\mathcal{T}_{\mathrm{PL}}^{(i)}}^{\mathrm{PL}, \mathcal{T}^{(i)}}\left|t_{\text {final }}^{P L}-t_{\text {initial }}^{P L}\right|\right) t_{\text {final }}^{P L}+ \\
\left.\left.\frac{\left(\left(\beta_{\mathcal{T}_{\mathrm{PL}}^{(i)}}^{\mathrm{PL}, \mathcal{T}^{(i)}}\left|t_{\text {final }}^{P L}-t_{\text {initial }}^{P L}\right| t_{\text {final }}^{P L}\right)^{2}\right.}{2}+\cdots\right)\right) \\
C_{\max _{i}},
\end{gathered}
$$

for a parasitic load which polynomially increases with respect to time. The rate of decrease that this load poses on the $C_{\max }$ value after consecutive flights is useful in being able to model different rates of decay in the circuit in stage $\mathcal{T}^{(i)}$ of the flight.

Step 5 (SOC formulas based on maximum capacitance formalism for the regular and local cases): From expressions of $C_{\max }$ and $\mathcal{C}^{P L}$, we can make use of the formula for computing the SOC to propose 3 different formulas that can be used to compute the corresponding state of charge, each of which are of the form, 


$$
\begin{aligned}
& \mathrm{SOC}_{1}=1-\frac{q_{\mathrm{max}}-q_{b}}{e^{\sum_{i}-\beta_{i} \times t_{\text {initial }}^{(i)}-t_{\text {final }}^{(i)} C_{\max }}}, \\
& \mathrm{SOC}_{2}=1-\left(q_{\max }-q_{b}\right) /\left[e^{\sum_{i}-\beta_{i}\left|t_{\text {initial }}^{(i)}-t_{\text {final }}^{(i)}\right|}\right. \\
& \left.\times e^{\beta_{\tau_{\mathrm{PL}}^{\mathrm{PL}, \mathcal{T}^{(i)}}}^{(i)}\left|t_{\text {final }}^{P L}-t_{\text {initial }}^{P L}\right|} C_{\max }\right], \\
& \mathrm{SOC}_{3}=1-\left(q_{\max }-q_{b}\right) /\left[1+\left(\beta_{\mathcal{T}_{\mathrm{PL}}^{(i)}}^{\mathrm{PL}, \mathcal{T}^{(i)}}\right.\right. \\
& \left(t_{\text {final }}^{P L}-t_{\text {initial }}^{P L}\right) t_{\text {final }}^{P L} \\
& +\frac{1}{2}\left(\beta_{\mathcal{T}_{\mathrm{PL}}^{(i)}}^{\mathrm{PL}, \mathcal{T}^{(i)}}\left|t_{\text {final }}^{P L}-t_{\text {initial }}^{P L}\right|\right. \\
& \left.\left.\left.t_{\text {final }}^{P L}\right)^{2}+\cdots\right) C_{\max }\right] \text {, }
\end{aligned}
$$

respectively, which together correspond to the SOC for the first formula for the capacitance introduced the earliest in the report, while the remaining 2 formulas below the first equation correspond to expressions for the SOC due to the local capacitance $\mathcal{C}$ of the parasitic load, with either exponential or polynomial rates of decay.

Step 6 (summarizing the procedure: backtracking from $t_{\text {abort }}$ to determine time intervals for which the current satisfies $30 \%$ SOC): From either a flight or parasitically driven mechanical abnormality, we can make flight predictions by following previous steps, in which exponentially weighing the duration of each flight, which poses mechanical or thermal stress on the engine, provides convenient interpretations for the total duration of a flight before the circuit fails to satisfy a specified SOC threshold.

\subsection{Rate of decay of the local capacitance due to a parasitic load}

As suggested through computations in previous tables, we are able to readily generate predictions for upcoming flights by specifying the stage of the flight at which the load is imposed, from which computations of the local capacitance, due to either polynomial or exponential decay, can be computed. Thus, from such a rate of decay, it is possible to establish suggestions for the maximum flight time given the duration of each stage of the flight. Conversely, past flight data from previous mechanical malfunctions can also be interpreted, as follows. If the duration of either a mechanical malfunction or a flight anomaly is known, the magnitude of the parasitic load, whether polynomial or exponential, can be determined by working backwards from the expressions given in the previous section. Namely, if the distance over which the mechanical or flight plan anomaly is known through experimentally gathered current measurements, then the distance over which the anomaly has occurred can be used to approximate the magnitude of the parasitic load responsible for the anomaly.

Furthermore, because an exponentially varying parasitic load that increases with respect to time can be Taylor expanded into a polynomial of fixed degree, computing the distance over which a flight anomaly have occurred may also be implemented to obtain a polynomial expression for the parasitic load that is responsible for the anomaly. In either circumstance, the approach fits within experimental data that is to be gathered in upcoming chamber experiments, or to data that has already been gathered, to inform maintenance of future flights. Clearly, if the magnitude due to the parasitic load is sufficiently dominant, in the sense that the degradation due to the parasitic load prevents the flight from completing individual stages of a flight that are of the same duration from flights that it has already completed previously in a given sequence, then the flight should be terminated.

\section{Concluding Remarks}

By introducing an exponentially decaying factor that is temporally weighed in specific sets of loading current versus time measurements that are experimentally recorded for flight patterns, we are able to generate widely accommodating flight predictions that take into account the specific nature of the data, which in this case consists of current drawn from the batteries supplied to the electric power-train throughout the flight, which is variable and can variably degrade with repeated use. In future work, we are continuing to pursue our interest of being able to continue generating sufficiently large combinatorial spaces from the $\Phi$ assignment. Although such a mapping is defined so that we do not assign multiple $\beta$ free parameters to the $C_{\max }$ decay for time periods of a flight that are not equal, there are countably many mappings that can be constructed to further experiment with the steepness of the $C_{\max }$ decay. Varying the rate of the $C_{\max }$ decay could be of additional interest for aircraft that are either built according to different specifications than the one in which HIRF chamber experiments were held. The developed methodology and the approach presented here is capable of addressing such changes in machinery by being able to explore families of $\Phi$ mappings, within the space of mappings $\Phi_{\mathcal{S}}$, where $\mathcal{S}$ is the sample space of times during individual stages of the flight. With a more general notion of the rate of change of the maximum capacitance with respect to not only the flight number of a cycle but also with respect to the hardware specific requirements of the battery and equipment that are being used, our approach can be of use in future experiments as well. 
Another area of interest for future work includes being able to develop a broader formalism so that the approach can describe other data structures that could arise in the experimentally gathered current readings. With more complicated experiments and specifications imposed upon either the amount of current that will be delivered throughout the circuit during a flight, or upon the duration of the flight itself, it would be helpful to continue expanding on the approach so that flights with an arbitrary number of stages can be analyzed, which could improve battery maintenance, as well as overall construction of the aircraft for optimal aerodynamic performance in a wide variety of flight profile patterns. Although the estimates for the decay in the maximum capacitance will not be subject to electromagnetic radiation effects that are present in the background of the environment which can significantly impact the temperature at which the equipment optimally functions, another exponential factor with its own free parameter accounting for the difference between the ambient temperature of the environment, and the maximum temperature at which the battery will continue to carry sufficient enough current above the SOC threshold, can be introduced.

\section{REFERENCES}

Goebel, K., \& Eklund, N. (2007). Prognostic fusion for uncertainty reduction. In Aiaa infotech@aerospace 2007 conference and exhibit.

Hogge, E., \& et. al. (2014). Verification of a remaining flying time predictions system for small elec- tric aircraft. In Annual conference of the phm society.

Kulkarni, C., Biswas, G., Celaya, J. R., \& Goebel, K. (2011). Prognostics techniques for capacitor degradation and health monitoring. In The maintenance and reliability conference, marcon.

Kulkarni, C., Biswas, G., Koutsoukos, X., Goebel, K., \& Celaya, J. (2010). Physics of failure models for capacitor degradation in dc-dc converters. In The maintenance reliability conference, marcon 2010 the maintenance \& reliability conference, marcon 2010.

Kulkarni, C., Hogge, E., Quach, C., \& Goebel, K. (2015). "hirf battery data set". In Nasa ames prognostics data repository. NASA.

Saha, B., Saha, S., \& Goebel, K. (2009). A distributed prognostic health management architecture. In Ieee aerospace conference.

Sankararaman, S., \& Goebel, K. (2013a). Remaining useful life estimation in prognosis: An uncertainty propagation problem. In Aiaa infotech conference.

Sankararaman, S., \& Goebel, K. (2013b). Uncertainty quantification in remaining useful life of aerospace components using state space models and inverse form. In $54^{\text {th }}$ aiaa structures, structural dynamics \& materials conference. 